\title{
Erratum to: Optimal adaptive sampling recovery
}

\section{Dinh Dũng}

Published online: 6 October 2011

(c) Springer Science+Business Media, LLC 2011

\section{Erratum to: Adv Comput Math (2011) 34:1-41 DOI 10.1007/s10444-009-9140-9}

We correct the definitions of the quantities of optimal sampling recovery $e_{n}(W)_{q}$ and $r_{n}(W)_{q}$ which have been introduced in [1]. All the results and proofs of [1] are unchanged and hold true for the new corrected definitions given below.

Recall that the definition of sampling recovery method $S_{n}^{B}$ was given by (1.4) in [1]. The worst case error of the recovery by $S_{n}^{B}$ for the function class $W$, is measured by

$$
\sup _{f \in W}\left\|f-S_{n}^{B}(f)\right\|_{q} .
$$

Given a family $\mathcal{B}$ of subsets in $L_{q}$, we consider optimal sampling recoveries by $B$ from $\mathcal{B}$ in terms of the quantity

$$
R_{n}(W, \mathcal{B})_{q}:=\inf _{B \in \mathcal{B}} \inf _{S_{n}^{B}} \sup _{f \in W}\left\|f-S_{n}^{B}(f)\right\|_{q} .
$$

We assume a restriction on the sets $B \in \mathcal{B}$, requiring that they should have, in some sense, a finite capacity. The capacity of $B$ is measured by its cardinality or pseudo-dimension. This reasonable restriction would provide nontrivial lower bounds of asymptotic order of $R_{n}(W, \mathcal{B})_{q}$ for well known function classes $W$.

Communicated by Charles Micchelli.

The online version of the original article can be found under doi:10.1007/s10444-009-9140-9.

D. Dũng $(\bowtie)$

Information Technology Institute, Vietnam National University,

Hanoi 144 Xuan Thuy, Cau Giay, Hanoi, Vietnam

e-mail: dinhdung@vnu.edu.vn 
Denote $R_{n}(W, \mathcal{B})_{q}$ by $e_{n}(W)_{q}$ if $\mathcal{B}$ in (1) is the family of all subsets $B$ in $L_{q}$ such that $|B| \leq 2^{n}$, where $|B|$ denotes the cardinality of $B$, and by $r_{n}(W)_{q}$ if $\mathcal{B}$ in (1) is the family of all subsets $B$ in $L_{q}$ of pseudo-dimension at most $n$.

Let $\Phi=\left\{\varphi_{k}\right\}_{k \in J}$ be a family of elements in $L_{q}$. Denote by $\Sigma_{n}(\Phi)$ the nonlinear set of linear combinations of $n$ free terms from $\Phi$, that is $\Sigma_{n}(\Phi):=\{\varphi=$ $\left.\sum_{j=1}^{n} a_{j} \varphi_{k_{j}}: k_{j} \in J\right\}$. The quantity $s_{n}(W, \Phi)_{q}$ which has been introduced in $[1,2]$ (denoted by $v_{n}(W, \Phi)_{q}$ in [2]), can be equivalently redefined as

$$
s_{n}(W, \Phi)_{q}:=\inf _{S_{n}^{B}: B=\Sigma_{n}(\Phi)} \sup _{f \in W}\left\|f-S_{n}^{B}(f)\right\|_{q} .
$$

A different definition of $s_{n}(W, \Phi)_{q}$ is as follows. For each function $f \in W$, we choose a sequence $\left\{x^{s}\right\}_{s=1}^{n}$ of $n$ points in $\mathbb{I}^{d}$. This choice defines $n$ sampled values $\left\{f\left(x^{s}\right)\right\}_{s=1}^{n}$. Then we choose a sequence $a=\left\{a_{s}\right\}_{s=1}^{n}$ of $n$ numbers and a sequence $\left\{\varphi_{k_{s}}\right\}_{s=1}^{n}$ of $n$ functions from $\Phi$, depending on the sampling information of $\left\{x^{s}\right\}_{s=1}^{n}$ and $\left\{f\left(x^{s}\right)\right\}_{s=1}^{n}$. This choice defines a sampling recovery method given by

$$
A_{n}^{\Phi}(f):=\sum_{s=1}^{n} a_{s} \varphi_{k_{s}}
$$

Notice that the set of all sampling recovery methods $A_{n}^{\Phi}$ coincides with the set of all $S_{n}^{B}$ such that $B=\Sigma_{n}(\Phi)$. Therefore, there holds true the equality

$$
s_{n}(W, \Phi)_{q}=\inf _{A_{n}^{\Phi}} \sup _{f \in W}\left\|f-A_{n}^{\Phi}(f)\right\|_{q},
$$

where the infimum is taken over all sampling recovery methods $A_{n}^{\Phi}$ of the form (2). Hence, we can take (3) as an alternative definition of $s_{n}(W, \Phi)_{q}$.

It is easy to verify that for the above corrected and new definitions, there hold true the inequalities $e_{n}(W)_{q} \geq \varepsilon_{n}(W)_{q}, r_{n}(W)_{q} \geq \rho_{n}(W)_{q}$ and $s_{n}(W, \Phi)_{q} \geq$ $\sigma_{n}(W, \Phi)_{q}$ which were used in $[1,2]$ for the proofs the lower bounds of $e_{n}\left(U_{p, \theta}^{\alpha}\right)_{q}, r_{n}\left(U_{p, \theta}^{\alpha}\right)_{q}$ and $s_{n}\left(U_{p, \theta}^{\alpha}, \mathbf{M}\right)_{q}$.

\section{References}

1. Dũng, D.: Optimal adaptive sampling recovery. Adv. Comput. Math. 34, 1-41 (2011)

2. Dũng, D.: Non-linear sampling recovery based on quasi-interpolant wavelet representations. Adv. Comput. Math. 30, 375-401 (2009) 